\title{
Validation of Functional Assessment of Cancer Therapy with Nasopharyngeal Cancer Subscale (FACT-NP) for Quality of Life in Thai Patients
}

\author{
Thanarpan Peerawong, M.D. ${ }^{1}$, Chanon Kongkamol, M.D. M.Sc. ${ }^{2}$, Zhiping Chen, M.D. ${ }^{3}$, \\ Paytai Rordlamool, M.D. ${ }^{1}$, Duangjai Sangtawan, M.D. ${ }^{1}$, Rungarun Jiratrachu, M.D. ${ }^{1}$ \\ ${ }^{1}$ Department of Radiology, ${ }^{2}$ Research Unit of Holistic Health and Safety Management in the Community, \\ Faculty of Medicine, Prince of Songkla University, Hat Yai, Songkhla 90110, Thailand. \\ ${ }^{3}$ Department of Radiotherapy, Cancer Hospital of Jiangxi Province, Qing ShanHu District Nanchang, Jiangxi, 330029, China. \\ Received 3 December 2018 • Revised 4 June 2019 • Accepted 15 June 2019 • Published online 9 July 2019
}

\section{Abstract:}

Objective: To develop and validate the Thai version of the functional assessment of cancer therapy with nasopharyngeal cancer subscale (FACT-NP) with the abbreviated version of the World Health Organization Quality of Life-BREF instrument (WHOQOL-BREF).

Material and Methods: A cross-sectional study was performed in the Radiology Department between January 2014 and October 2016. Inclusion criteria: nasopharyngeal carcinoma patients aged greater than 18 years. Exclusion criteria: no comprehension of the Thai language, had other cancers (except for skin cancer and diagnosed with impaired cognition and/or overt psychosis), major depression or delirium. After signing the consent form, participants were interviewed and self-completed FACT-NP and WHOQOL-BREF. The structure of the FACT-NP was determined with exploratory factors analysis. The internal reliability of identified domains was evaluated using Cronbach's alpha. The correlation between domains of the modified FACT-NP and the domains of WHOQOL-BREF were examined with Spearman's correlation. Known-group validity was determined by comparing patients with different sociodemographic and clinical characteristics using the T-test, Ranksum test and analysis of variance. A p-value of less than 0.05 was considered significant.

Results: This study was terminated early due to a long accrual period. Of the 230 patients included in the study, only 220 completed both FACT-NP and WHOQOL-BREF. Exploratory factor analysis showed an accumulative variance

Contact: Asst. Prof. Thanarpan Peerawong, M.D.

Department of Radiology, Faculty of Medicine, Prince of Songkla University,

Hat Yai, Songkhla 90110, Thailand.

E-mail: pthanarpan@gmail.com
J Health Sci Med Res 2019;37(...):................ doi: 10.31584/jhsmr.201958 www.jhsmr.org 
of 0.56 with 4 factors. The internal reliability of modified FACT-NP was 0.92 . There was moderate correlation between modified FACT-NP and WHOQOL-BREF. Both the modified FACT-NP and WHOQOL-BREF could identify differences between the groups.

Conclusion: The Thai modified FACT-NP was found to be both valid and reliable for measuring the quality of life in Thai nasopharyngeal cancer patients.

Keywords: FACT-NP, factor analysis, nasopharyngeal cancer, quality of life

\section{Introduction}

Nasopharyngeal cancer is a subset of head and neck cancers for which radiotherapy is a form of treatment that can cure the patient. This disease is rare in Western countries but more common in Southeast Asia. Incidence of nasopharyngeal cancer in Thailand is fifth in Asia. ${ }^{1}$ Nowadays, combined radiotherapy and chemotherapy can improve progression-free survival and overall survival. It has become the standard treatment in patients with or without metastatic disease. ${ }^{2-4}$ The treatments depend on the stage of the disease. In the early stages nasopharyngeal carcinoma is treated with radical radiotherapy. In more advanced cases, combined radiotherapy and chemotherapy are prescribed. Generally, a total radiation dose of 70 gray in 35 fractions is prescribed. A total of 5 cycles of chemotherapy are given, according to the protocol of the institute. Cisplatin 100 milligram per square meter $\left(\mathrm{mg} / \mathrm{m}^{2}\right)$ or carboplatin area under curve (AUC) 6 is given every 3 weeks for 3 cycles in the concurrent phase. After radiation is completed, an additional 2 cycles of cisplatin $75-80 \mathrm{mg} / \mathrm{m}^{2}$ or carboplatin AUC5 on day 1 and 5-fluorouracil (5-FU) $1,000 \mathrm{mg} / \mathrm{m}^{2}$ on days $1-4$ every 4 weeks are administered. Doses of chemotherapy are adjusted based on patient hematological results and renal toxicity. Generally, radiotherapy causes patients to suffer from complications such as chronic dysphagia, xerostomia, and fibrosis of the neck. Additional chemotherapy is associated with higher levels of severe hearing loss. ${ }^{5}$
Quality of life (QOL) is a broad multidimensional concept that usually includes subjective evaluations of both the positive and negative aspects of life. ${ }^{6}$ Healthrelated quality of life (HRQOL) instruments have been developed to measure and classify generic HRQOL, disease-specific quality of life, and cancer-specific quality of life. Disease specific questionnaires may be more sensitive to detect differences when compared with generic quality of life questionnaires, which do not focus on the issues of particular concern to patients with a disease. $^{7}$ Measuring quality of life in nasopharyngeal cancer: there are 2 common systems available, the functional assessment of cancer therapy with nasopharyngeal cancer subscale (FACT-NP) and the head and neck module (H\&N35) from the European Organization for Research and Treatment of Cancer Quality of Life. Although H\&N35 is widely used, this questionnaire was developed for all head and neck cancers. ${ }^{8}$ In contrast, FACT-NP was developed specifically for nasopharyngeal cancer. Thus, this questionnaire focuses on evaluating the suffering caused by treatment toxicities as a result of nasopharyngeal cancer treatment. This is the questionnaire we chose for the study.

To the best of our knowledge, no nasopharyngeal cancer specific questionnaire has been validated in the Thai language. This study aimed to develop and validate the Thai version of the FACT-NP for measuring quality of life, and compare it with the World Health Organization 
Quality of Life-BREF (WHOQOL-BREF) in nasopharyngeal cancer patients.

\section{Material and Methods}

\section{Ethical consideration}

The study was approved by the Human Research and Ethics Committee of the Faculty of Medicine, Prince of Songkla University, REC: 56-006-07-1-3

\section{Study design and setting}

The cross-sectional study was performed in the largest radiation-oncology unit in southern Thailand, which serves approximately 2,500 new radiotherapy patients per year. The enrollment period was between January 2014 and October 2016.

\section{Study samples}

Nasopharyngeal carcinoma patients aged more than 18 years who visited the hospital for treatment or follow-up were included in the study. Those who did not understand the Thai language, had other cancers (except for skin cancer and diagnosed with impaired cognition and/or overt psychosis), major depression or delirium were excluded.

\section{Instruments}

FACT-NP is the functional assessment of cancer therapy general (FACT-G) with nasopharyngeal subscale. The researchers collaborated with the Functional Assessment of Chronic Illness Therapy Organization on the translation. The translation, back translation, and linguistic validation process were performed using the Functional Assessment of Chronic IIIness Therapy translation project guidelines. The final version of the Thai FACT-NP was pilot tested with 10 treated nasopharyngeal carcinoma patients using an interview script. We did not find any misunderstandings in the translation.
FACT-NP comprises 43 items with a 5-point (0-4: not at all to very much) Likert scale and category to 5 domains: physical well-being (PWB), social/family wellbeing (SWB), emotional well-being (EWB), functional well-being (FWB) and nasopharyngeal cancer subscales (NPS). The ranges of the scores of these domains are 0-28, $0-28,0-24,0-28$ and $0-64$, respectively. The range of total score for FACT-NP is $0-172$. Higher scores mean a higher QOL.

WHOQOL-BREF was validated in the Thai language with radiotherapy patients. ${ }^{9,10}$ The questionnaire comprises 26 items with a 5-point (1-5: not at all to very much) Likert scale and was categorized into 4 domains: physical health $(\mathrm{PH})$, psychological well-being (PSW), social relationship (SR) and satisfaction with their environment (SE). The score of the subscale was calculated by summing the corresponding items in the subscale. The overall score ranged from 26-130. Higher scores mean a higher quality of life: categorized to bad (26-60), average (61-95) and good (96-130).

Independent variables were sociodemographic and clinical characteristics. Sociodemographic characteristics were age, religious, marital status, education level, and economic and working status. The clinical characteristics were clinical stage, Eastern Cooperative Oncology Group (ECOG) performance status, treatment and disease status, and history of percutaneous gastrostomy.

\section{Data collection}

All nasopharyngeal carcinoma patients who were eligible for the study were invited to participate. After signing the consent form, the research assistant interviewed them for sociodemographic data and reviewed their clinical characteristics. Next, patients answered the questionnaires by themselves. For the patients with reading difficulties, well-trained research assistants read aloud each item on the questionnaire and allowed the patients 
to choose his/her answer by himself or herself. Total time spent on these procedures was around 30 minutes.

\section{Statistical analyses}

The sociodemographic and clinical data were determined with descriptive statistics. In the development process, exploratory factor analysis (EFA) was used to group the items in FACT-G. The number of factors was determined with a scree plot, with the eigenvalue closest to the unity. Acceptable loading for each variable was $0.32 .{ }^{11}$ The oblimin was used in accordance with the previous study. ${ }^{12}$ The group of domains from EFA was checked for internal reliability using Cronbach's alpha. An alpha value of at least 0.7 was considered acceptable for internal consistency. If the alpha values were at least 0.8 and 0.9 , they were considered good and excellent for internal consistency, respectively. Spearman's correlation was calculated to determine the relationship between the domains of the modified FACT-NP and of WHOQOL-BREF for construct validity. The correlation coefficients ranged from -1 to +1 and were categorized into negligible correlation (0.00 to 0.30$)$, low correlation (0.3 to 0.5$)$, moderate correlation (0.5-0.7), high correlation (0.7-0.9), and very high correlation (0.9-1.0). Both scales were finally compared on their relationship with sociodemographic and clinical characteristics by t-test, Ranksum test and analysis of variance. A p-value of less than 0.05 was considered significant. Statistical analysis was conducted using the R program.

The sample size was calculated for testing the validity of the questionnaire by exploratory factor analysis. The adequate sample used was 1:5 item respondent ratio. $^{11}$ The total items of FACT-NP were 43 . Hence, the estimated sample size was at least 215 cases. An additional $15.0 \%$ was added. Thus, a total of 245 participants were included in the study.

\section{Results}

\section{Characteristics of the subjects}

The study was terminated early due to a long accrual period. A total of 230 patients were recruited for the study. However, only 220 patients completed both FACT-NP and WHOQOL-BREF. These 220 participants were used in data analysis. The majority was Buddhist, married and male with an age of around 50 years; most had no economic problems. Of the subjects, $57.7 \%$ were educated at least at the secondary school level. Most of the diagnosed stage III and IV nasopharyngeal cancer patients were treated with chemo-radiation and had a history of prophylactic gastrostomy. Of them, $37.7 \%$ were in the active treatment phase and $55.5 \%$ had no evidence of cancer recurrence or metastasis. The details are shown in the Table 1.

Table 1 Socio-demographic and clinical characteristic of participants

\begin{tabular}{ll}
\hline Variables & $\begin{array}{l}\text { Number (\%) } \\
(\mathbf{n}=220)\end{array}$ \\
\hline Age (mean and standard deviation) & $50.6 \pm 12.2$ \\
Male & $150(68.2)$ \\
Religious & \\
Buddhism & $190(86.4)$ \\
Islamism & $30(13.6)$ \\
Status & \\
Single & $26(11.8)$ \\
Married or couple & $183(83.2)$ \\
Divorce & $11(5.0)$ \\
Education level & \\
Bachelor and above & $59(26.8)$ \\
Secondary school & $68(30.9)$ \\
Primary school & $86(39.1)$ \\
Unlettered & $7(3.2)$ \\
Working & $127(57.7)$ \\
Economic problem & $45(20.5)$ \\
\hline
\end{tabular}


Table 1 (continued)

\begin{tabular}{ll}
\hline Variables & $\begin{array}{l}\text { Number (\%) } \\
(\mathbf{n}=220)\end{array}$ \\
\hline Stage & \\
I & $9(4.1)$ \\
III & $20(9.1)$ \\
IV & $86(39.1)$ \\
Eastern Cooperative Oncology Group performance status \\
$0-1$ & $105(47.7)$ \\
$2-4$ & $209(95.0)$ \\
Active treatment & $11(5.0)$ \\
Disease status & $83(37.7)$ \\
Loco-regional disease & \\
No evidence of disease & $70(31.8)$ \\
Recurrence or metastases & $122(55.5)$ \\
Treatment & $28(12.7)$ \\
Before treatment & \\
Radiation & $66(30.0)$ \\
Chemo-radiation & $14(6.4)$ \\
Supportive treatment & $138(62.7)$ \\
Prophylactic gastrostomy & $2(0.9)$ \\
\hline
\end{tabular}

\section{Exploratory factor analysis}

The Kaiser-Meyer-Olkin of sampling adequacy was 0.87 and Bartlett's test of sphericity was significant with $p$-value $<0.001$, which shows these data were suited for factor analysis. Figure 1 revealed a relationship between eigenvalue and factor number. Then the number of factors in EFA was 4. The results of EFA are shown in Table 2. The factor loading in each domain from 1, 2, 3 and 4 was $0.17,0.13,0.13$ and 0.13 . The cumulative variance was 0.56. The new factors had the same names as the previous FACT-NP, which were social and family wellbeing, emotional well-being, physical well-being and functional well-being, respectively.

The Cronbach's alpha in each domain and the total items of FACT-NP ranged from 0.82-0.92. The details are shown in Table 3. The internal consistency of each domain was good or excellent.

Parallel analysis scree plots

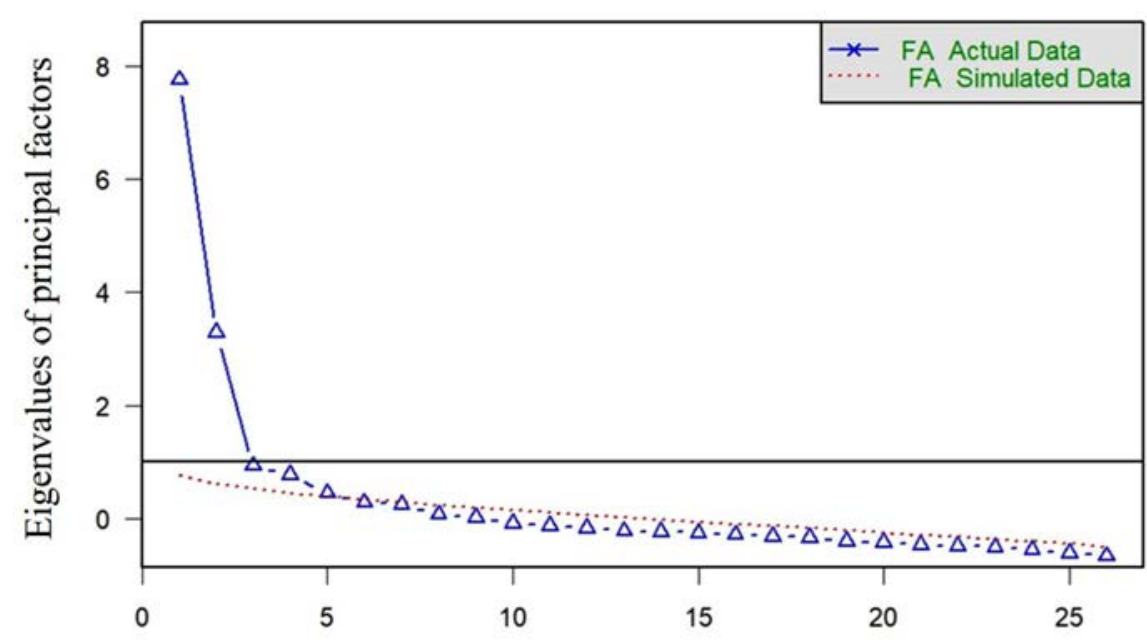

Factor number

Figure 1 Relationship between number of factor and eigenvalue 
Table 2 The exploratory factor analysis of the modified functional assessment of cancer therapy general in relation to factor structure and loadings

\begin{tabular}{|c|c|c|c|c|c|c|c|}
\hline \multirow{2}{*}{ Factor structure originally } & \multicolumn{7}{|c|}{ Component in this analysis } \\
\hline & 1 & 2 & 3 & 4 & h2 & u2 & com \\
\hline GP1 I have a lack of energy & & 0.70 & & & 0.60 & 0.40 & 1.1 \\
\hline GP2 I have nausea & & 0.40 & & & 0.25 & 0.75 & 1.6 \\
\hline GP4 I have pain & & 0.62 & & & 0.45 & 0.55 & 1.1 \\
\hline GP6 I feel ill & & 0.77 & & & 0.67 & 0.33 & 1.1 \\
\hline GP7 I am forced to spend time in bed & & 0.73 & & & 0.57 & 0.43 & 1.1 \\
\hline GS1 I feel close to my friends & 0.57 & & & & 0.45 & 0.55 & 1.5 \\
\hline GS2 I get emotional support from my family & 0.78 & & & & 0.69 & 0.31 & 1.1 \\
\hline GS3 I get support from my friends & 0.61 & & & & 0.45 & 0.55 & 1.2 \\
\hline GS4 My family has accepted my illness & 0.82 & & & & 0.72 & 0.28 & 1 \\
\hline GS5 I am satisfied with family communication about my illness & 0.91 & & & & 0.80 & 0.20 & 1 \\
\hline GS6 I feel close to my partner (or the person who is my main support) & 0.77 & & & & 0.60 & 0.40 & 1 \\
\hline GS7 I am satisfied with my sex life & 0.55 & & & & 0.45 & 0.55 & 1.3 \\
\hline GE1 I feel sad & & & 0.56 & & 0.52 & 0.48 & 1.6 \\
\hline GE2 I am satisfied with how I am coping with my illness & & & & 0.33 & 0.38 & 0.62 & 2.4 \\
\hline GE3 I am losing hope in the fight against my illness & & & 0.65 & & 0.56 & 0.44 & 1.1 \\
\hline GE4 I feel nervous & & & 0.85 & & 0.75 & 0.25 & 1 \\
\hline GE5 I worry about dying & & & 0.84 & & 0.70 & 0.30 & 1.1 \\
\hline GE6 I worry that my condition will get worse & & & 0.49 & & 0.39 & 0.61 & 1.5 \\
\hline GF2 My work (include work at home) is fulfilling & & -0.44 & & 0.45 & 0.47 & 0.53 & 2.2 \\
\hline GF3 I am able to enjoy life & & & & 0.85 & 0.72 & 0.28 & 1 \\
\hline GF4 I have accepted my illness & & & & 0.74 & 0.67 & 0.33 & 1.2 \\
\hline GF5 I am sleeping well & & & & 0.44 & 0.41 & 0.59 & 1.8 \\
\hline GF6 I am enjoying the things I usually do for fun & & & & 0.66 & 0.61 & 0.39 & 1.1 \\
\hline GF7 I am content with the quality of my life right now & & & & 0.54 & 0.54 & 0.46 & 1.4 \\
\hline Cumulative variance 0.56 & 0.17 & 0.13 & 0.13 & 0.13 & & & \\
\hline
\end{tabular}

GP=general physical, GS=general social, GE=general emotional, GF=general functional, h2= communality, u2=uniqueness, com=Hoffmann's item complexity 
Table 3 Scoring method and Cronbach's alpha coefficients of modified functional assessment of cancer therapy with nasopharyngeal subscale

\begin{tabular}{llll}
\hline Subscales & Items & $\begin{array}{l}\text { Score } \\
\text { range }\end{array}$ & $\begin{array}{l}\text { Cronbach's } \\
\text { alpha }\end{array}$ \\
\hline Physical well-being & 5 & $0-28$ & 0.82 \\
Social and family well-being & 7 & $0-28$ & 0.88 \\
Emotional well-being & 5 & $0-24$ & 0.85 \\
Functional well-being & 7 & $0-28$ & 0.87 \\
Nasopharyngeal cancer & 16 & $0-64$ & 0.86 \\
subscales & & & \\
\hline Total score & 40 & $0-172$ & 0.92 \\
\hline
\end{tabular}

\section{Validity}

Convergent validity: the details of correlation between the domains in the modified FACT-NP and the WHOQOL-BREF are shown in Figure 2. The PWB and EWB had a low positive correlation with each domain in WHOQOL-BREF, but a 0.41 in the $\mathrm{PH}$ domain. The SWB had a moderate correlation with the SR and SE domains. The FWB had a moderate positive correlation with each domain of WHOQOL-BREF. The NPS had a low positive correlation with $\mathrm{PH}$ and PSW.

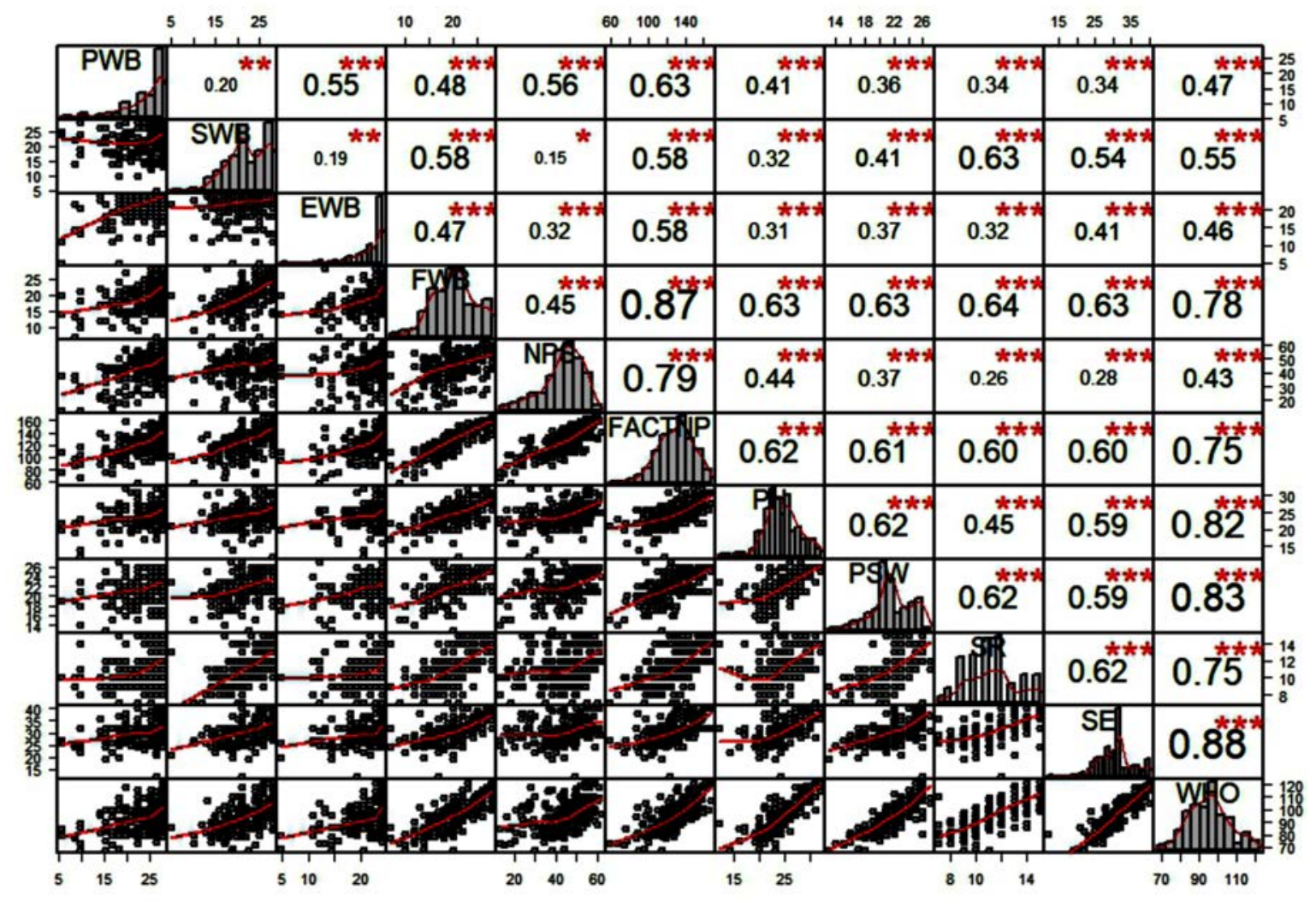

The statistic calculated by Spearman's correlation.

${ }^{*} p-$ value $\leq 0.05,{ }^{* *} p-$ value $\leq 0.01,{ }^{* *} p-$ value $\leq 0.001, P W B=$ physical well-being, $S W B=$ social and family well-being, EWB=emotional wellbeing, FWB=functional well-being, NPS=nasopharyngeal cancer subscales, FACT-NP=functional assessment of cancer therapy with nasopharyngeal cancer subscale, $\mathrm{PH}=$ physical health, PSW=phychological well-being, SR=social relationship, SE=satisfaction with the environment and $\mathrm{WHO}=$ overall score of abbreviated version of World Health Organization Quality of Life

Figure 2 Correlation matrix between domain in modified functional assessment of cancer therapy with nasopharyngeal cancer subscale and abbreviated version of World Health Organization Quality of Life. 
In the known group validity, the discrimination pattern between WHOQOL-BREF and modified FACT-NP in patient characteristics was similar. The details are shown in Table 4.

Table 4 The quality of life score of abbreviated version of World Health Organization Quality of Life and modified functional assessment of cancer therapy with nasopharyngeal cancer subscale classified by patient charectoristics $(n=220)$

\begin{tabular}{|c|c|c|c|c|}
\hline \multirow{3}{*}{ Patient characteristics (\%) } & \multicolumn{4}{|c|}{ Quality of life score } \\
\hline & \multicolumn{2}{|c|}{ WHOQOL-BREF } & \multicolumn{2}{|c|}{ Modified FACT-NP } \\
\hline & Mean (S.D.) & $p$-value & Mean (S.D.) & $p$-value \\
\hline \multicolumn{5}{|l|}{ Age (years) } \\
\hline$\geq 60(20.0)$ & $94.2(13.7)$ & 0.421 & $125.9(20.1)$ & 0.559 \\
\hline$<60(80.0)$ & $95.9(11.4)$ & & $128(21.3)$ & \\
\hline \multicolumn{5}{|l|}{ Working } \\
\hline Yes (57.7) & $98.5(11.7)$ & $<0.001$ & $134.1(19.2)$ & $<0.001$ \\
\hline No (42.3) & $91.5(10.9)$ & & $118.6(20.1)$ & \\
\hline \multicolumn{5}{|l|}{ Economic problem } \\
\hline Yes (20.5) & $92.2(11.6)$ & 0.031 & 119 (23.3) & 0.002 \\
\hline No (79.5) & $96.4(11.8)$ & & $129.7(19.9)$ & \\
\hline \multicolumn{5}{|l|}{ Stage } \\
\hline I (4.1) & $98.2(14.4)$ & 0.011 & $132(16.1)$ & 0.002 \\
\hline ॥ $(9.1)$ & $98.2(10.1)$ & & $136.2(19.3)$ & \\
\hline III (39.1) & $98(12.1)$ & & $131.9(21.9)$ & \\
\hline IV $(47.7)$ & $92.8(11.3)$ & & $122(19.7)$ & \\
\hline \multicolumn{5}{|l|}{ ECOG performance status } \\
\hline $0-1(95.0)$ & $96.1(11.8)$ & 0.003 & $129.2(20.1)$ & $<0.001$ \\
\hline $2-4(5.0)$ & $85.4(8.1)$ & & $97(14.6)$ & \\
\hline \multicolumn{5}{|l|}{ Active treatment } \\
\hline Yes (37.7) & $92.1(10.5)$ & $<0.001$ & $121(108.9,134.1)^{\star}$ & $<0.001$ \\
\hline No (62.3) & $97.6(12.2)$ & & $134(118.8,148)^{*}$ & \\
\hline \multicolumn{5}{|l|}{ Disease status } \\
\hline Loco-regional disease (31.8) & $91.8(10.5)$ & $<0.001$ & $122.4(18.5))$ & $<0.001$ \\
\hline No evidence of disease (55.5) & $98.8(11.7)$ & & $134.2(19.1)$ & \\
\hline Recurrence or metastases (12.7) & $90.8(11.8)$ & & $111.4(23.1)$ & \\
\hline \multicolumn{5}{|l|}{ Prophylactic percutaneous gastrostomy } \\
\hline Yes $(67.1)$ & $96.3(11.8)$ & 0.172 & $129.3(20.9)$ & 0.069 \\
\hline No (32.9) & $94.0(11.9)$ & & $123.9(20.8)$ & \\
\hline \multicolumn{5}{|l|}{ Quality of life } \\
\hline Good (51.4) & $102(98,110)^{*}$ & $<0.001$ & $141(132,152)^{*}$ & $<0.001$ \\
\hline Average (48.6) & $86(82,91)^{*}$ & & $(104.2,125.3)^{*}$ & \\
\hline
\end{tabular}

*Median (interquartile range), S.D.=standart deviation, WHOQOL-BREF=abbreviated version of World Health Organization Quality of Life, FACT-NP=functional assessment of cancer therapy with nasopharyngeal cancer subscale, ECOG=Eastern Cooperative Oncology Group, The statistic calculated by Ranksum test, t-test and ANOVA F-test 


\section{Discussion}

The Thai version of FACT-NP is a nasopharyngeal cancer quality of life questionnaire. It has 39 items and includes 5 domains: PWB, SWB, EWB, FWB and NPS. The questionnaire has excellent internal consistency. Although the construct validity and convergent validity were shown to have a low to moderate correlation with the domain in WHOQOL-BREF, the pattern of differentiation by socioeconomic and clinical characteristic between modified FACT-NP and WHOQOL-BREF is similar.

There had been no developed and validated study of FACT-NP in other languages before. Pattern of factor analysis in this study followed Ding et al.'s study ${ }^{13}$, which performed factor analysis only in the FACT-G part and added the cervical cancer subscale to functional assessment of cancer therapy with cervical cancer subscale (FACT-CX). The cumulative variances were 0.5 . In the validation of Thai FACT-G, which was mainly performed in breast cancer patients, it had cumulative variances of 0.56. The factor loading pattern was not the same as the original version of FACT-G. The items GS1: "I feel close to my friends" and GS3: "I get support from my friends" were loaded in FWB instead of SWB. The item GE2: "I am satisfied with how I am coping with my illness" was loaded in FWB instead of EWB. ${ }^{12}$ Compared to our study, the cumulative variance is not different. The factor loading in our study had the same pattern as in the Thai version of FACT-G. However, the items were reduced to 24 . The GF1: "I am able to work (includes work at home)" had singularity with GF2: "My work (includes work at home) is fulfilling". The other items were dropped during factor analysis. For the total score of FACT-NP, although some items were dropped, our study calculated the total score with reference to the original version. The score in each domain in the modified version will be changed to the original version scale by proportion. Then the total score can be compared to the original FACT-NP in the future.
The reliability of modified FACT-NP is, at least, good. In the original version of FACT-NP, Cronbach's alpha in each domain ranged from $0.84-0.90$ and 0.95 for total items. In the FWB domain, the original version had excellent internal consistency, but was only good in the modified version. ${ }^{14}$ When compared to the validated Thai FACT-G study, Cronbach's alpha in each domain ranged from 0.75-0.87. The internal consistency was acceptable in SWB and good in the others. ${ }^{12}$ Our internal consistency result was good, although there were fewer items.

In the structural evaluation by convergent validity: The modified Thai FACT-NP had a high positive correlation with WHOQOL-BREF. But in the domains it had a low to moderate correlation to WHOQOL-BREF. This result was explained by $\mathrm{Yu}$ et al. $^{15}$ who performed the validation study of FACT-G in Hong Kong. The results also showed a low correlation between WHOQOL-BREF and FACT-G study. The original version of FACT-NP evaluated convergent validity by using the correlation between FACT-NP and the Quality of Life-Radiation Therapy Instrument-Head and Neck companion module (QOL-RTH\&N which was a cancer specific questionnaire). The original FACT-NP had a high positive correlation with total QOL-RT-H\&N score. However, in general, QOL and socioeconomic domains had a high positive correlation and moderate correlation with the FWB domain, respectively. The functional and head-and-neck domains had a high positive correlation with the NPS domain. The emotional domain also had a moderate positive correlation with NPS domain. ${ }^{14}$ These results explain that the quality of life questionnaire did not measure the same aspects of general health as the cancer specific questionnaire. Thus, carefully consideration is necessary when choosing the questionnaire.

Although the discriminative pattern between modified FACT-NP and WHOQOL-BREF were the same, the difference of QOL scores in financial burden, treatment 
status and disease stage results in our study were also similar to the original Thai FACT-G. ${ }^{12}$ Two previous prospective randomized studies showed that prophylaxis percutaneous gastrostomy in head and neck cancer improved 6-month QOL after treatment. From 173 cases, there were only 2 cases of nasopharyngeal carcinoma patients in the reactive gastrostomy arm. ${ }^{16,17}$ The benefit of prophylactic percutaneous gastrostomy in nasopharyngeal carcinoma is unknown. The modified FACTNP showed that patients who have undergone prophylactic percutaneous gastrostomy tend to have better QOL. These results indicate that general health related quality of life, disease specific quality of life, and cancer specific quality of life had a common ability to differentiate patients with financial burdens, treatment status, and disease stage. The specific issues which influence QOL in nasopharyngeal cancer, such as dry mouth, hearing loss and dysphagia, were not included in this study. The benefits of the cancer specific quality of life questionnaire could not be seen in the study. Modified FACT-NP is an option in quality of life measurement in nasopharyngeal cancer. The FACT-NP is available on https://www.facit.org.

There are some limitations: First, this study was a cross-sectional study. The other properties of the cancer specific questionnaire, such as responsiveness were not determined. Second, type of radiation therapy technique was not considered in the study. Radiation technique influences nasopharyngeal cancer patient QOL, although many studies have shown that intensity modulated radiotherapy technique (IMRT) can reduce radiation toxicities with better QOL outcomes than the conventional or threedimension radiotherapy technique. ${ }^{18-20}$ At the time of this study, IMRT was not routinely performed in nasopharyngeal cancer patients in our institution.

\section{Conclusion}

The Thai modified FACT-NP was found to be both valid and reliable for measuring quality of life in nasopharyngeal carcinoma patients.

\section{Acknowledgement}

We would like to thank all the staff members in the Radiation Oncology Unit, Department of Radiology, Faculty of Medicine, Prince of Songkla University for their kind support. The study was financially supported by the Faculty of Medicine, Prince of Songkla University.

\section{Conflict of interest}

The authors declare that there is no conflict of interest regarding the publication of this article.

\section{References}

1. Ferlay J, Ervik M, Lam F, Colombet M, Mery L, Piñeros M, et al. Global cancer observatory: cancer today [homepage on the Internet]. Lyon: International Agency for Research on Cancer; 2018. [cited 2018 Feb 04]. Available from: https://gco.iarc.fr/ today

2. Yan M, Kumachev A, Siu LL, Chan KK. Chemoradiotherapy regimens for locoregionally advanced nasopharyngeal carcinoma: a Bayesian network meta-analysis. Eur J Cancer 2015;51:1570-9.

3. Zhang L, Zhao C, Ghimire B, Hong MH, Liu Q, Zhang Y, et al. The role of concurrent chemoradiotherapy in the treatment of locoregionally advanced nasopharyngeal carcinoma among endemic population: a meta-analysis of the phase III randomized trials. BMC Cancer 2010;10:558.

4. National Comprehensive Cancer Network (NCCN). NCCN Clinical practical guidelines in oncology. Head and neck cancers (Version 2.2018) [homepage on the Internet]. Pennsylvania: NCCN; 2018 [cited 2018 Apr 14]. Available from: https://www. nccn.org/professionals/physician_gls/pdf/head-andneck.pdf 
5. Du C, Ying HM, Kong FF, Zhai RP, Hu CS. Concurrent chemoradiotherapy was associated with a higher severe late toxicity rate in nasopharyngeal carcinoma patients compared with radiotherapy alone: a meta-analysis based on randomized controlled trials. Radiat Oncol 2015;10:70.

6. The Whogol Group. The World Health Organization Quality of Life Assessment (WHOQOL): development and general psychometric properties. Soc Sci Med 1998;46:1569-85.

7. Fayers PM, Machin D. Introduction. In: Fayers PM, Machin D. editors. Quality of life: the assessment, analysis, and interpretation of patient-reported outcomes. $2^{\text {nd }}$ ed. West Susex: Wiley; 2007

8. Singer S, Arraras JI, Chie WC, Fisher SE, Galalae R, Hammerlid $\mathrm{E}$, et al. Performance of the EORTC questionnaire for the assessment of quality of life in head and neck cancer patients EORTC QLQ-H\&N35: a methodological review. Qual Life Res 2013;22:1927-41.

9. Mahatnirunkul S, Pumpisanchai W, Tuntipivatanakul W. Comparison of the WHOQOL-100 and the WHOQOL-BREF (26 items). J Ment Health Thai 1998;5:4-15.

10. Phungrassami T, Katikarn R, Watanaarepornchai S, Sangtawan D. Quality of life assessment in radiotherapy patients by WHOQOL-BREF-THAI: a feasibility study. J Med Assoc Thai 2004;87:1459-65.

11. Anna BC, Jason WO. Best practices in exploratory factor analysis: four research \& evaluation. PARE 2005;10:1-9

12. Ratanatharathorn V, Sirilerttrakul S, Jirajarus M, Silpakit C, Maneechavakajorn J, Sailamai P, et al. Quality of life, functional assessment of cancer therapy-general. J Med Assoc Thai 2001; 84:1430-42.

13. Ding $Y, H u Y$, Hallberg IR. Psychometric properties of the chinese version of the functional assessment of cance therapy-cervix (FACT-Cx) measuring health-related quality of life. Health Qual Life Outcomes 2012;10:124.
14. Tong MC, Lo PS, Wong $\mathrm{KH}$, Yeung RM, van Hasselt CA, Eremenco S, et al. Development and validation of the functional assessment of cancer therapy nasopharyngeal cancer subscale. Head Neck 2009;31:738-47.

15. Yu CL, Fielding R, Chan CL, Tse VK, Choi PH, Lau WH, et al. Measuring quality of life of Chinese cancer patients: a validation of the Chinese version of the Functional Assessment of Cancer Therapy-General (FACT-G) scale. Cancer 2000;88: 1715-27.

16. Salas S, Baumstarck-Barrau K, Alfonsi M, Digue L, Bagarry $\mathrm{D}$, Feham N, et al. Impact of the prophylactic gastrostomy for unresectable squamous cell head and neck carcinomas treated with radio-chemotherapy on quality of life: prospective randomized trial. Radiother Oncol 2009;93:503-9.

17. Silander E, Nyman J, Bove M, Johansson L, Larsson S, Hammerlid E. Impact of prophylactic percutaneous endoscopic gastrostomy on malnutrition and quality of life in patients with head and neck cancer: a randomized study. Head Neck 2012;34:1-9.

18. Huang TL, Chien CY, Tsai WL, Liao KC, Chou SY, Lin HC, et al. Long-term late toxicities and quality of life for survivors of nasopharyngeal carcinoma treated with intensitymodulated radiotherapy versus non-intensity-modulated radiotherapy. Head Neck 2016;38(Suppl 1):E1026-32.

19. Lastrucci L, Bertocci S, Bini V, Borghesi S, De Majo R, Rampini A, et al. Late toxicity, evolving radiotherapy techniques, and quality of life in nasopharyngeal carcinoma. Radiol Med 2017; 122:303-8.

20. Pan XB, Huang ST, Chen KH, Jiang YM, Ma JL, Qu S, et al. Intensity-modulated radiotherapy provides better quality of life than two-dimensional conventional radiotherapy for patients with stage II nasopharyngeal carcinoma. Oncotarget 2017; 8:46211-8. 